\title{
DÜBLIN
}

Technological University Dublin

ARROW@TU Dublin

2014-01-01

\section{Diagnostics of an 02-He RF Atmospheric Plasma Discharge by Spectral Emission}

\author{
Vladimir Milosavljevic \\ Technological University Dublin, vladimir.milosavljevic@tudublin.ie \\ Mick Donegan \\ University College Dublin \\ Patrick Cullen \\ Technological University Dublin, pj.cullen@tudublin.ie
}

See next page for additional authors

Follow this and additional works at: https://arrow.tudublin.ie/schfsehart

Part of the Atomic, Molecular and Optical Physics Commons, and the Plasma and Beam Physics Commons

\section{Recommended Citation}

Milosavljevic, V., Donegan, M., Cullen, P.J. and Dowling, D.P. Diagnostics of an 02-He RF Atmospheric Plasma Discharge by Spectral Emission. Journal of the Physical Society of Japan 83, 014501 (2014). doi.org/10.7566/JPSJ.83

This Article is brought to you for free and open access by the School of Food Science and Environmental Health at ARROW@TU Dublin. It has been accepted for inclusion in Articles by an authorized administrator of ARROW@TU Dublin. For more information, please contact arrow.admin@tudublin.ie, aisling.coyne@tudublin.ie, gerard.connolly@tudublin.ie.

Funder: the Department of Agriculture, Food and the Marine, Ireland

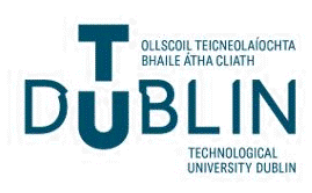


Authors

Vladimir Milosavljevic, Mick Donegan, Patrick Cullen, and Denis Dowling

This article is available at ARROW@TU Dublin: https://arrow.tudublin.ie/schfsehart/132 


\title{
Diagnostics of an $\mathrm{O}_{2}-\mathrm{He}$ RF Atmospheric Plasma Discharge by Spectral Emission
}

\author{
Vladimir Milosavljevici ${ }^{1,2,3,4 *}$, Mick Donegan ${ }^{5}$, Patrick J. Cullen ${ }^{1}$, and Denis P. Dowling ${ }^{5}$ \\ ${ }^{1}$ BioPlasma Research group, Dublin Institute of Technology, Sackville Place, Dublin 1, Ireland \\ ${ }^{2}$ Biosystems Engineering, University College, Dublin, Dublin 4, Ireland \\ ${ }^{3}$ NCPST, Dublin City University, Dublin, Ireland \\ ${ }^{4}$ Faculty of Physics, University of Belgrade, P.O.B. 368, Belgrade, Serbia \\ ${ }^{5}$ School of Mechanical and Materials Engineering, University College Dublin, Belfield, Dublin 4, Ireland
}

(Received March 12, 2013; accepted October 25, 2013; published online xxxx yy, zzzz)

In this paper optical emission spectroscopy (OES) is used as a Diagnostic technique for the measurement of atomic and molecular spectral emissions generated using a helium if industrial atmospheric plasma jet system. The OES of neutral atomic spectral lines and molecular bands are investigated over a range of plasma process parameters. Wavelength resolve optical emission profiles suggest that the emission of helium's spectral lines shows that the high energy electrons have a larger influence than helium metastables on the overall spectral emission. Furthermore, the experimental data indicates that the use of high helium flow rates, in any confined open air plasma discharge, limits the significance of air impurities, e.g., nitrogen, for the creation and sustainability of plasma discharges in helium-oxygen gas chemistry.

\section{Introduction}

Atmospheric pressure, non-thermal helium plasma jets are ${ }_{4}$ increasingly used in many processing applications, due to 25 their combination of inherent plasma stability and excellent 26 reaction chemistry, which is often enhanced downstream of 27 the plasma source. The majority of the atmospheric pressure ${ }_{28}^{8}$ plasmas are non-equilibrium at ambient temperature and 29 are generated by electrical discharge. Despite their wide30 spread usage, it remains largely unknown whether cold 31 atmospheric plasma jets maintain similar characteristics, such 32 as gas temperatures and particle flux, when they breakdown 33 while arcing or whether they possess different operating 34 modes.

Therefore, in order to ensure process reproducibility, the 30 monitoring and control of plasma processes is essential in ${ }_{37}$ both laboratory and industrial environments. Most plasma 38 systems, other than specialised laboratory plasma systems, do 39 not facilitate intrusive plasma diagnostics (i.e., for collecting 40 local plasma properties) or have plasma chambers with only 4) one or two access points. It is also important to underline, 42 that due to the high industrial demand for plasma 43 technologies and the resulting competition between system 44 manufacturers, many of these manufacturers come to the 45 market with closed box plasma systems (typically a plasma 46 generator and its matching network in the single box), which ${ }_{47}$ cannot be opened due to warranty issues. This leads to the 48 situation where the only plasma diagnostic techniques 49 available are optical techniques. Under these circumstances 50 it is very challenging to develop an experimental approach, 51 which would give a fundamental explanation of the impact of 52 plasma physics on atomic physics, e.g., the role of metastable 53 atoms, resonance energy levels, triplet and singlet energy 54 scale, life time of electrons in an excited atom/molecular 55 state, etc. One non-intrusive technique that has been ${ }_{56}$ successfully used for plasma diagnostics is optical emission 67 spectroscopy (OES). A disadvantage of the OES technique is ${ }_{58}$ that the integration of measured signals must be carried out 59 over a line of sight observation. Despite this limitation ${ }_{60}$ however, OES is commonly used to detect light emitted by ${ }_{61}$ excited species from plasma discharges.
The type of operating gas influences the stability of 21 atmospheric plasma discharges. Helium gives rise to a stable 22 homogeneous discharge, whereas nitrogen, oxygen and argon 23 easily cause the transition into a filamentary discharge. ${ }^{1)}{ }_{24}$ Atmospheric plasma, propagating in the surrounding ambient 25 air, can incur impurities due to the surrounding ambient 26 atmosphere, which also follows the feed gas channel. The ${ }_{27}$ most dominant species in the atmosphere is nitrogen. ${ }^{2)}$ In the 28 vast majority of atmospheric plasma discharges, nitrogen ${ }_{29}$ dominates the ionic composition of atmospheric discharge 30 and has an impact on the breakdown voltage. When nitrogen ${ }_{31}$ is added/mixed with helium plasma discharges, the helium ${ }_{32}$ emission lines are significantly quenched and the resulting ${ }_{33}$ plasma changes from a reddish colour to strong green. In the ${ }_{34}$ $300-450 \mathrm{~nm}$ spectral range, the strongest emission is the $\mathrm{N}_{2}{ }^{+}{ }_{35}$ first negative system $\left(\mathrm{B}^{2} \Sigma_{\mathrm{u}}{ }^{+} \rightarrow \mathrm{X}^{2} \Sigma_{\mathrm{g}}{ }^{+}\right)$and the $\mathrm{N}_{2}$ second ${ }_{36}$ positive system $\left(C^{3} \Pi_{u} \rightarrow B^{3} \Pi_{g}\right)$. On the other hand, nitro- ${ }^{37}$ gen in most atmospheric plasma is not a carrier gas and ${ }_{38}$ therefore can dilute the plasma chemistry from the feed gas 39 channel, acting as a contaminant gas. Moreover as nitrogen 40 comes from the outside of the discharge channel and is 41 always at ambient temperature, it effects the temperature of 42 the plasma discharge. ${ }^{3)}$ There is also an important role for ${ }_{43}$ nitrogen in the generation of $\mathrm{NO}_{x}$ (and other greenhouse ${ }_{44}$ gases), acid rain and other environmental problems. There- 45 fore minimizing the influence of nitrogen, as an unwanted 46 addition, to plasma discharges in the ambient air is an 47 important challenge. This is especially true because the 48 plasma chemical conversion of $\mathrm{N}_{2}$ and $\mathrm{O}_{2}$ into $\mathrm{NO}_{x}$ is a very 49 efficient process. ${ }^{4}$ )

Our main motivation in the present work is (1) to 51 investigate the influence of high-energy electrons and helium 52 metastables on the overall spectral emission, (2) to study 53 how high helium flow rates, in a confined open air plasma 54 discharge, limits the significance of air impurities in the 55 creation and sustainability of plasma discharges in the 56 helium-oxygen gas chemistry, (3) to minimize the influence 57 of nitrogen, as an unwanted contaminant to plasma 58 discharges in the ambient air, thus limiting the important 59 role of nitrogen in the generation of greenhouse gases etc., 60 (4) to explore the production channel of oxygen radicals by 61 
direct electron impact (excitation process) from the ground 2 state of the $\mathrm{O} I$ in the high helium flow rate plasma dis3 charge.

\section{Experimental}

Included in this work is the use of OES for recording the absolute spectral emissions of the atomic and molecular lines associated with helium, oxygen, nitrogen and hydrogen. 9 Analysis of these species spectral intensities will assist in the development of optimised plasma processing parameters for treatments such as polymer surface activation, the removal of contaminates etc.

\subsection{Helium spectral emission}

Helium has, as it is well known, two independent sets of energy levels (singlet and triplet), and an ionization limit of $1724.59 \mathrm{eV}^{5}$ ) There are triplet and singlet excited energy levels 18 in a neutral helium atom (He I) and electron transition among 19 these are forbidden in dipole-dipole approximations. In 20 general, the intercombination of spontaneous transition 21 probabilities from triplet to singlet for neutral helium is very ${ }_{22}$ low. Therefore both of the spectral emissions (from the triplet ${ }_{23}$ and singlet energy levels), should be taken into account ${ }_{24}$ separately for a valid representation of a full helium spectral 25 radiation. The intensity of helium was monitored over six 26 prominent atomic helium spectral lines. There are three 27 spectral lines from the helium triplet spectra $\mathrm{He} 388$ ( $\mathrm{He} \mathrm{I}$; 2s $\left.\lambda=388.865 \mathrm{~nm} ; \quad 2 \mathrm{~s}^{3} \mathrm{~S}_{1}-3 \mathrm{p}^{3} \mathrm{P}_{1,2}^{\circ}\right), \quad H e 587 \quad(\mathrm{He} \mathrm{I} ; \lambda=$ ${ }_{29} 587.562 \mathrm{~nm} ; 2 \mathrm{p}^{3} \mathrm{P}_{1,2}^{\mathrm{o}}-3 \mathrm{~d}^{3} \mathrm{D}_{1,2,3}$ ) and He706 (He I; $\lambda=$ 30 $706.519 \mathrm{~nm} ; 2 \mathrm{p}^{3} \mathrm{P}_{1,2}^{0}-3 \mathrm{~s}^{3} \mathrm{~S}_{1}$ ). There are also three spectral ${ }_{31}$ lines from the helium singlet spectra He501 (He I; ${ }_{32} \lambda=501.568 \mathrm{~nm} ; \quad 2 \mathrm{~s}^{1} \mathrm{~S}_{0}-3 \mathrm{p}^{1} \mathrm{P}_{1}^{\mathrm{o}}$ ), He667 (He I; $\lambda=$ 33 $667.815 \mathrm{~nm} ; 2 \mathrm{p}^{1} \mathrm{P}_{1}^{\mathrm{o}}-3 \mathrm{~d}^{1} \mathrm{D}_{2}$ ) and He728 (He I; $\lambda=$ $\left.{ }_{34} 728.135 \mathrm{~nm} ; 2 \mathrm{p}^{1} \mathrm{P}_{1}^{\mathrm{o}}-3 \mathrm{~s}^{1} \mathrm{~S}_{0}\right)$. ${ }^{5)}$ Helium's high metastable en35 ergy levels, which act as a "reservoir of energy", make it 30 ideal for use for plasma processing. ${ }^{1,6)}$ Density of helium ${ }_{37}$ metasables atoms can be measured by laser absorption ss spectroscopy $^{7)}$ and the results of these experiments are 39 dependant on helium gas flow rate. The measurement of $\mathrm{He}$ 40 metasable densities in Ref. 7 have been performed for two ${ }_{41}$ plasma systems, one of which (the mesh-type), is similar to ${ }_{42}$ the plasma jet in this work. The results presented for the ${ }_{43}$ mesh-type system ${ }^{7}$ ) indicate that at atmospheric pressure 44 helium metasable densities decrease with increasing gas flow ${ }_{45}$ rate. Moreover, Fig. 5 (in Ref. 7) indicates that helium higher 46 flow rates quench the excited species, especially the long${ }_{47}$ lived metasable atoms, causing a decrease in the discharge 48 current with increasing gas flow rates. On the helium triplet 49 energy scale, there is a metastable energy level at $1 \mathrm{~s} 2 \mathrm{~s}\left({ }^{3} \mathrm{~S}_{1}\right)$ 50 with an energy of $19.82 \mathrm{eV}$. Using OES, this energy level can 51 be observed as the He388 spectral line emission and therefore 52 the spectral intensity of this atomic line can be used for the 53 monitoring of a metastable helium atom in the triplet state. ${ }_{54}$ The $1 \mathrm{~s} 2 \mathrm{~s}\left({ }^{1} \mathrm{~S}_{0}\right)$ is a metastable energy level in the helium ${ }_{55}$ singlet state with energy of $20.62 \mathrm{eV}$. The lower energy level ${ }_{56}$ of $\mathrm{He} 501$ is $2 \mathrm{~s}\left({ }^{1} \mathrm{~S}_{0}\right)$. Therefore the spectral intensity of the 57 $\mathrm{He} 501$ is proportional to the metastable density of helium 58 singlet atoms. Four additional helium spectral lines, dis59 cussed in this work, all have lower energy levels, which are ${ }_{60}$ the same as the helium resonate levels. They are He587 and ${ }_{61} \mathrm{He} 706$ lines which each have lower energy level $1 \mathrm{~s} 2 \mathrm{p}\left({ }^{3} \mathrm{P}_{1}^{\circ}\right)$ with the energy of $20.96 \mathrm{eV}$, and this is the resonate level of , the helium triplet state. The He667 and He 728 have the lower 2 energy level $1 \mathrm{~s} 2 \mathrm{p}\left({ }^{1} \mathrm{P}_{1}^{\circ}\right)$ with a energy of $21.22 \mathrm{eV},{ }^{5)}$ and this 3 energy level is a resonant level of the helium singlet state. 4 The lifetime of electrons in resonate energy levels are much 5 shorter than the lifetime in an "ordinary" excited energy 6 level ${ }^{5)}$ and is many orders of magnitude shorter than an 7 electrons life time at metastable energy levels. Because of 8 this the quenching of resonate energy levels is negligible and 9 therefore an electron transition that ends on a resonate energy 10 level may be use for the monitoring of an atom's density in " its ground state.

\subsection{Oxygen spectral emission}

Oxygen species were studied in the He plasma using 15 measurements of atomic $(\mathrm{O} \mathrm{I})$ and molecular $\left(\mathrm{O}_{2}\right)$ spectral 16 emissions. All recorded atomic oxygen spectral lines are 17 triplets. $^{5)}$ The following atomic oxygen triplet spectral 18 lines are recorded: 0615 (O I; $\lambda=615.598,615.677$, 18 and $\left.615.818 \mathrm{~nm} ; 3 \mathrm{p}^{5} \mathrm{P}_{1,2,3}-4 \mathrm{~d}^{5} \mathrm{D}_{1,3,4}^{\circ}\right), 0777$ (O I, $\lambda={ }_{20}$ $777.194,777.417$, and $\left.777.539 \mathrm{~nm} ; 3 \mathrm{~s}^{5} \mathrm{~S}_{2}^{\circ}-3 \mathrm{p}^{5} \mathrm{P}_{1,2,3}\right)$ and ${ }_{21}$ O845 (O I, $\lambda=844.625,844.636$, and $844.676 \mathrm{~nm} ; 22$ $3 s^{3} \mathrm{~S}_{1}^{\circ}-3 \mathrm{p}^{3} \mathrm{P}_{0,1,2}$ ). The $\mathrm{O} 615$ spectral emission comes from ${ }_{23}$ the high laying excited energy level of $12.75 \mathrm{eV}^{5)}$ The lower 24 energy level of the 0615 triplet coincides with the upper 25 states of the 0777 triplet, with an energy of $10.74 \mathrm{eV}$. 26 Therefore the O615 triplets increase in intensity emission, 27 would result in a change of the relative intensity of the 28 individual lines of the 777 triplet, and this could lead to errors 29 in using the spectral intensity of 777 triplet for plasma ${ }_{30}$ diagnostics, since the one of 0777 spectral line is used for ${ }_{31}$ actinometry. ${ }^{8)}$ The third recorded atomic oxygen spectral line, 32 O845, has the energy threshold of the excited states of ${ }_{33}$ $10.99 \mathrm{eV}^{5)}$ The $\mathrm{O} 615, \mathrm{O} 777$, and $\mathrm{O} 845$, are created by direct 34 excitation from a ground state, but could be also created over 35 a $\mathrm{O}_{2}$ dissociation. The energy threshold for $\mathrm{O}_{2}$ dissociation, 36 and selected spectral lines, are: $16.1 \mathrm{eV}$ (O777), $16.3 \mathrm{eV}_{37}$ (O845), and $18.0 \mathrm{eV}(\mathrm{O} 615)$. These energies are lower than 38 the energy of helium metastables, therefore the dissociation 39 of molecular oxygen must be taken into account in the 40 interpretation of atomic oxygen spectral line intensity.

The neutral oxygen molecule does not readily show an 42 emission spectrum, as the spectral emissions of molecular 43 oxygen generally have very weak spectral emissions, 44 however these can be observed under optimised atmospheric 45 pressure conditions. The A-band $\left(\mathrm{O}_{2} 760\right)$ is the only recorded 46 molecular oxygen emission in this work. The A-band has 47 a band-head emission at $\lambda=759.37 \mathrm{~nm}$ and belongs to a 48 transition $b^{1} \Sigma_{g}{ }^{+}-X^{3} \Sigma_{g}{ }^{-9}$.9) Oxygen molecules have seven 49 long-living metastable states located in the energy diagram 50 below the first dissociation limit, at approximately $5.1 \mathrm{eV}$. 51 The upper energy state $\left(\mathrm{b}^{1} \Sigma_{\mathrm{g}}{ }^{+}\right)$of the $\mathrm{O}_{2} 760$ emission is one ${ }_{52}$ of molecular oxygen metastables with energies of $1.63 \mathrm{eV} .53$ Transition from metastable to the ground state only occurs 54 in a magnetic dipole transition (singlet-triplet intercombina- 55 tion). Because of that the A-band has a low transition 56 probability and its upper energy level has a relatively long 57 life time (approx. $7 \mathrm{~s}$ ). The big advantage of the $\mathrm{b}^{1} \Sigma_{\mathrm{g}}{ }^{+}$is its 58 energy $(1.63 \mathrm{eV})$ which is within the average energy range 59 of electrons in most electrical discharges (low-pressure or 60 atmospheric). ${ }^{10)}$ 
Water vapor is present in many atmospheric plasma 2 discharges in ambient air. Thus, oxygen as a part of a 3 molecule could also be recorded over $\mathrm{H}_{2} \mathrm{O}$ and/or the $\mathrm{OH}$ 4 spectral emissions. In this work, only the $\mathrm{OH}$ emission is 5 recorded. The $\mathrm{OH}$ radical with $\mathrm{Q}_{1}$ band-head at $\lambda=$ $308.986 \mathrm{~nm}(\mathrm{OH} 309)$ and transition $\mathrm{A}^{2} \Sigma^{+}(v=0)-\mathrm{X}^{2} \Pi$ $>\left(v^{\prime}=0\right)$ has been recorded in all experimental conditions. a The OH309 emission can be used for a thermal analysis of the plasma. The rotational temperature was measured from intensities of components of the $Q_{1}$ branch of the $(0,0)$ band 11 for the electronic transition $\mathrm{OH}$ radical as it is close to the 12 gas temperature. ${ }^{11,12)}$ The upper energy level of $\mathrm{OH} 309$ is 13 metasable with an energy of $4.17 \mathrm{eV}$. The ionization energy ${ }_{14}$ of the $\mathrm{OH}$ molecule $(13.18 \mathrm{eV})$ is just slightly lower than the 15 ionization energy of a neutral hydrogen $(13.62 \mathrm{eV})$ and both ${ }_{16}$ species $(\mathrm{OH}$ and $\mathrm{H})$ are produced through $\mathrm{H}_{2} \mathrm{O}$ dissociation.

\section{${ }_{18} 2.3$ Hydrogen and nitrogen spectral emissions}

Atomic hydrogen $(\mathrm{H} \mathrm{I})$ is studied using the emission of the Balmier- $\alpha$ line $(\lambda=656.279 \mathrm{~nm} ; n=2-3)$. The upper 2 energy level of the Balmier- $\alpha$ line has an energy of $12.09 \mathrm{eV}$. ${ }_{22}$ The lower energy level $(10.20 \mathrm{eV})$ of the Balmier- $\alpha$ line is 23 a resonant energy level in atomic Hydrogen. This resonate ${ }_{24}$ level has the highest transition probability, i.e., the shortest ${ }_{25}$ lifetime, in a hydrogen atom. Thus, quenching of the $n=2$ 20 atomic hydrogen energy level is most unlikely. A spectral ${ }_{27}$ intensity of the Balmier- $\alpha$ line could therefore be used for the 20 density estimation of atomic hydrogen.

Nitrogen spectra are almost always found to be present in open air electrical discharges. In this research, one of the goals is to minimise the nitrogen spectral emission by optimizing the experiment. The only recorded nitrogen emission, in this work, is the second positive system with ${ }^{34}$ the band-head at $\lambda=337.14 \mathrm{~nm}$ and transition $\mathrm{C}^{3} \Pi_{\mathrm{u}}$ ${ }_{35}(v=0)-\mathrm{B}^{3} \Pi_{\mathrm{g}}\left(v^{\prime}=0\right)$. This molecular nitrogen emission $36\left(\mathrm{~N}_{2} 337\right)$ is of frequent occurrence as an impurity in the 37 spectra of atmospheric plasma discharge. The upper and 38 lower energy levels of $\mathrm{N}_{2} 337$ spectral emission have energies 39 of 11.03 and $7.35 \mathrm{eV}$, respectively. The ionization energy of 40 $\mathrm{N}_{2}$ molecule is $15.58 \mathrm{eV}$.

\section{2.4 The setup}

${ }_{43}$ This OES study was carried out on the plasma jet formed 44 using the SurFx Atomflo ${ }^{\mathrm{TM}} 400 \mathrm{~L}$ atmospheric plasma ${ }_{45}$ system. This type of plasma source is widely used for the ${ }_{46}$ plasma treatment of polymers in order to enhance surface ${ }_{47}$ energy. ${ }^{12)}$ The source operates at a rf frequency of $27.12 \mathrm{MHz}$ 48 and forms a plasma $5 \mathrm{~cm}$ wide, using its $\mathrm{AH}-500 \mathrm{~L}$ beam 49 applicator (Fig. 1). The RF power is shown on the controller 50 of plasma source. The controller includes an RF generator 51 with a fast auto-tuning matching network. ${ }^{13)}$

Since it is very difficult to directly measure the output 3 power of the SurFx plasma source, due to its closed box ${ }^{4}$ design, it is useful to calculate the thermal energy transfer of 55 the plasma. Thermal imaging of a ceramic substrate, mounted 56 below the jet, was obtained using an InfraTec Vari CAM 57 high-resolution infrared thermographic camera and a K-type ${ }_{58}$ thermocouple. The measurement procedure builds on pre59 vious work, reported in literature. ${ }^{12,14)}$ An alumina ceramic ${ }_{60}(W) 21 \mathrm{~cm},(L) 27 \mathrm{~cm}$, and $(H) 0.4 \mathrm{~cm}$ was chosen as the ${ }_{61}$ substrate, as it has a high specific heat capacity $(850$

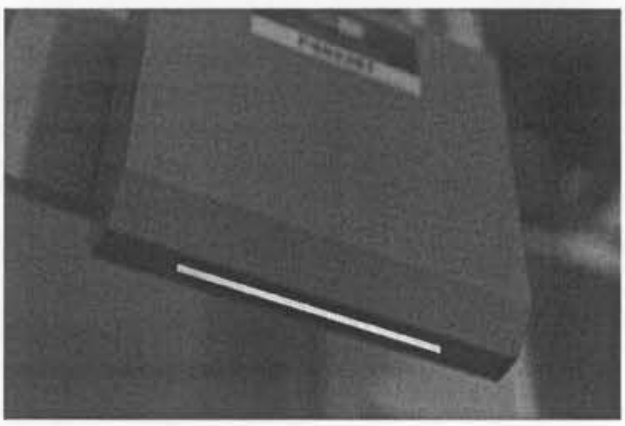

Fig. 1. (Color online) Photograph of SurFx Atomflo ${ }^{\mathrm{TM}}$ plasma source with 12 $5 \mathrm{~cm}$ long applicator orifice.

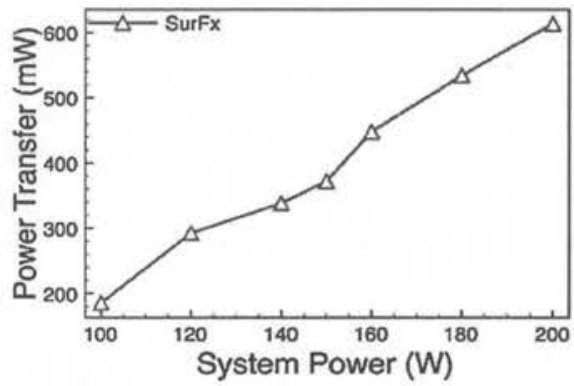

13

18

Fig. 2. Calculated thermal energy transfer values from the SurFx source to 26 a ceramic substrate, placed $16 \mathrm{~mm}$ below the plasma nozzle, at the indicated ${ }_{27}$ processing powers.

$\left.\left.\mathrm{J} \cdot \mathrm{g}^{-1} \cdot \mathrm{K}^{-1}\right) \cdot{ }^{14}\right)$ Using thermal imaging data, the thermal power 31 transfer $(P)$ can be estimated by measuring the surface ${ }_{32}$ temperature rise and the associated affected surface area. The ${ }_{33}$ data is then used to determine the time-dependent heating ${ }_{34}$ process using ${ }^{15)}$

$$
P=m \cdot C_{P} \cdot \frac{\Delta T}{\Delta t}
$$

In Eq. (1), $m$ represents the mass of the heated region, $C_{P}$ is specific heat capacity of the substrate $\left(\mathrm{J} \cdot \mathrm{g}^{-1} \cdot \mathrm{K}^{-1}\right)$, while 40 $\Delta T / \Delta t$ is the change in substrate temperature $(T$, in $\mathrm{K})$ in ${ }_{41}$ relation to the time ( $t$, in s). Thermal data was obtained while 42 the plasma applicator head was mounted $16 \mathrm{~mm}$ above the 43 ceramic surface. After ignition of the plasma, the change in 44 temperature of the ceramic's surface was recorded until a 45 steady state maximum temperature was reached. This was 46 repeated for input process powers in the range of 100-200 W 47 (as indicated in Fig. 2). Using the IRBIS 3 plus software 48 package, supplied with the thermal imaging camera, the 49 average temperature was calculated for a $1 \times 1 \mathrm{~cm}^{2}$ section of 50 the ceramic substrate, positioned directly under the plasma 51 jet, 15 and $30 \mathrm{~s}$ after the plasma was ignited. It should 52 be noted that some thermal energy will be lost to the 53 surrounding ambient in the $16 \mathrm{~mm}$ between the plasma 54 nozzle and the ceramic substrate and so the calculations 55 presented in this section do not represent the absolute 56 energies of the plasmas investigated. The differences in the 57 ceramic's temperature values (at 15 and $30 \mathrm{~s}$ ) were used as 58 the $\Delta T$ values in Eq. (1). The total mass of the $21 \times 27 \mathrm{~cm}^{2} 59$ ceramic substrate was measured to be $915 \mathrm{~g}$; the mass of the 60 $1 \mathrm{~cm}^{2}$ ceramic area treated was therefore calculated to be 61 
, $1.6 \mathrm{~g}$. Since the same time interval, of $15 \mathrm{~s}$, is used as the $\Delta t$ 2 function for all of the calculations, the thermal power transfer 3 is directly proportional to the change in temperature of the ${ }_{4}$ substrate. The thermal power transfer to the ceramic plate is 5 therefore calculated to be

$$
P=91.45 \cdot \Delta T \text {. }
$$

Figure 2 shows the thermal energy transfer from the plasmas formed using the SurFx plasma jet system. A to relatively linear relationship between system input power and 11 thermal energy transfer is observed. Thermal energy transfer 2 was in the range of $185-615 \mathrm{~mW}$, at the conditions 3 investigated.

The SurFx unit can generate plasma using $\mathrm{He}-\mathrm{O}_{2}$ gas mixtures (in the range of 100-200 W). The input variables for 16 this system are strictly limited by the equipment manufac7 turer, i.e., helium gas needs to flow at a constant flow rate of $301 / \mathrm{min}$, while oxygen gas input varies with plasma power. 19 The oxygen flow rate was maintained at less than $3 \%$ of the 20 helium flow rate at all times. Oxygen, which forms strong 21 oxidising agents once injected into a helium plasma, is used ${ }_{22}$ as the source of reactive species in this study. More details 23 about the experimental setup is presented in. ${ }^{12,16,17)}$ A feature ${ }_{24}$ of this source is the relatively contained plasma, which is 25 confined almost entirely inside the applicator housing. This 26 combined with the relatively high $\mathrm{He}$ flow rate maintains a 27 relatively un-contaminated plasma, despite its direct exposure 28 to the atmosphere.

Optical emission spectroscopy was carried out using a 30 low resolution USB4000 spectrometer. The OES technique is ${ }_{31}$ based on the integration of measured signals over a line of ${ }_{32}$ sight observation. Despite this limitation however, OES is s a non-intrusive diagnostic technique, which is commonly ${ }_{34}$ used to detect light emitted by excited species from plasma 35 discharges. Experiments were carried out to investigate 36 species intensity with varying power and time. ${ }^{12)}$ This 37 resulted in a matrix of 64 experiments. OES data is recorded 38 at the central point along the beam applicator, at $0,15,30,45$, ${ }_{39} 60$, and $75 \mathrm{~s}$ after the plasma was struck (i.e., the duration of a ${ }_{40}$ processing time), for all plasma powers. These OES experi"11 ments were repeated at two other points along the beam 42 applicator's orifice, as outlined in Ref. 12. A typical $\mathrm{He}-\mathrm{O}_{2}$ ${ }_{43}$ absolute intensity spectrum, recorded at $200 \mathrm{~W}$ after $30 \mathrm{~s}$, is ${ }_{44}$ shown in Fig. 3. The helium-oxygen gas velocity during this 45 experiment was calculated to be $6.9 \mathrm{~m} / \mathrm{s}$ and has a laminar 46 flow with a Reynolds number around $R e=158 .{ }^{18)}$ This ${ }_{47}$ figure also shows a helium spectrum recorded at a more 48 typical gas flow of $101 / \mathrm{min}$ (dashed line). The gas velocity 49 during this experiment was found to be $0.8 \mathrm{~m} / \mathrm{s}$ and flow was 50 found to be laminar with $R e=106 .{ }^{18)}$ It was established that 5113 different atomic and molecular spectral emission existed ${ }_{52}$ in the plasma. In order to truly establish species importance ${ }_{53}$ at the various processing parameters, it was necessary to 54 integrate the area under the emission peaks ${ }^{8)}$ for each OES ${ }_{55}$ spectra and to calculate a quantum efficiency of the spectral 56 system (the spectrameter, fiber optic cable, lens, etc.).

The area under the emission peaks shown on Fig. 3, were 58 measured and expressed in units of $\mathrm{mW} / \mathrm{cm}^{2}$ for a $1 \mathrm{~s}$ of 50 integration time. The low resolution of the spectrometer has 60 no influence on the profiles separation and the recorded o1 continuum emission is very low. There are small continuum

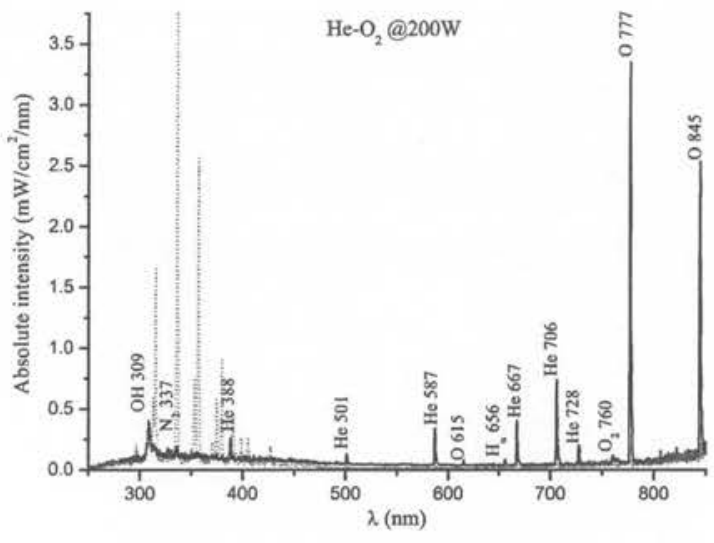

2
3
4
5
6
7
8
9
10
11
12
13

Fig. 3. (Color online) Spectrum of $\mathrm{He}-\mathrm{O}_{2}$ plasma discharge (solid line) 15 recorded $30 \mathrm{~s}$ after the plasma was struck. The importance of $\mathrm{OH} 309, \mathrm{~N}_{2} 337$, He388, etc. is discussed in Sects. 2.1-2.3. Also included is a typical helium plasma spectrum observed in an open air discharge (dashed line). ${ }^{18}$ )

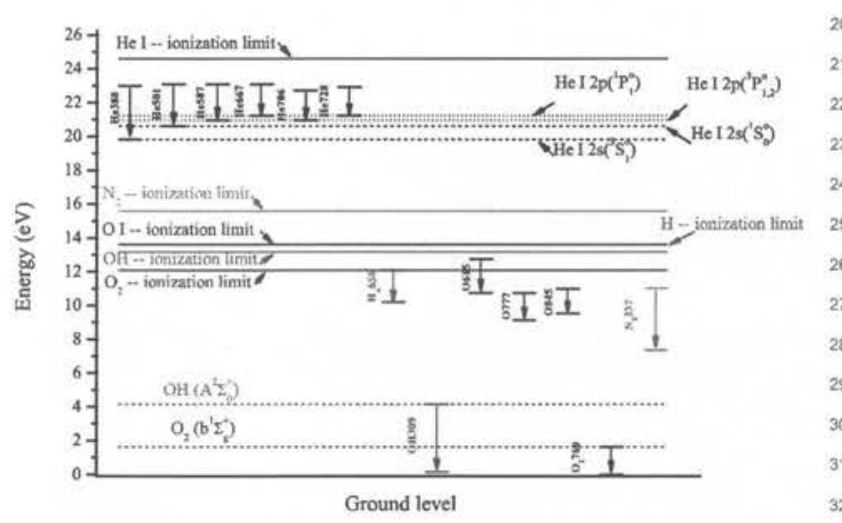

Fig. 4. (Color online) Energy diagram of the spectral emission from ${ }_{34}^{33}$ Fig. 3. All spectral line labels are the same as on Fig. 3.

emissions between 300 and $400 \mathrm{~nm}$, as well as at wave- 36 lengths higher than $800 \mathrm{~nm}$. The low continuum emission 39 indicates that gas temperature is not high, i.e., it is not 40 drastically different from the ambient temperature.

\section{Results and Discussion}

As well as optical spectra (Fig. 3), (nonradiative) energy 44 transfer among different plasma species is also important for 45 plasma diagnostics. The energy diagram in Fig. 4 takes into 46 account the energy levels of all spectral profiles from Fig. 3. 47

Figure 4 shows clear separation among helium energy 48 levels and the energy levels of other emitters in the plasma. 49 Helium has the highest excited energy states and metastable 50 levels and this needs to be taken into consideration when 51 other species are being created in the plasma.

The He emission at $706 \mathrm{~nm}$, with a threshold energy of ${ }_{53}$ $22.7 \mathrm{eV}$ and a radiative decay lifetime of the upper state of 54 $35 \mathrm{~ns}^{5}{ }^{5)}$ is an indicator of energetic electrons. ${ }^{19)}$ The He706 is 55 produced by radiative dissociation of the $\mathrm{He}_{2}{ }^{*}$ dimer, which 56 is formed by a recombination process from the metasable $\mathrm{He} 57$ atom. Even small amounts of nitrogen drastically change the 58 metasable helium density by fast Penning ionization with 59 $\mathrm{N}_{2} .{ }^{19)}$ Thus the intensity of the He706 decreases if the content 60 of air in the discharge is too high. Figure 5 shows the He706 61 


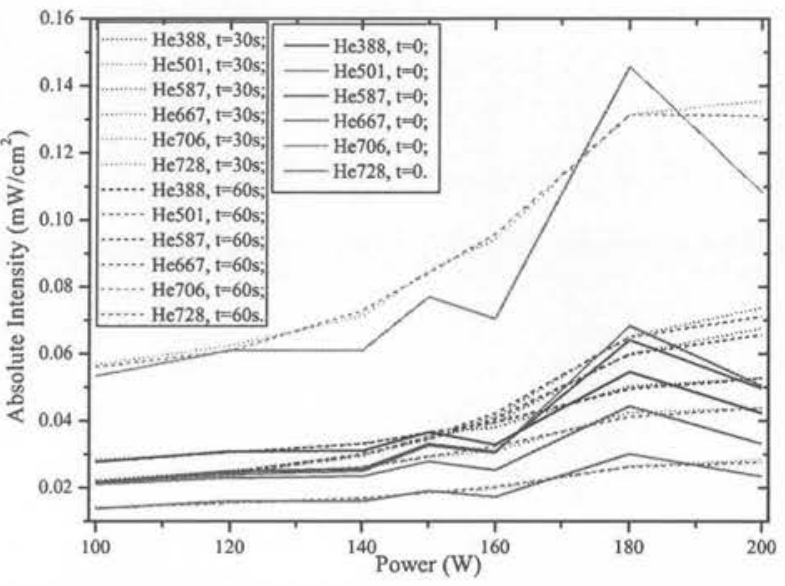

Fig. 5. (Color online) Absolute intensity of helium atomic spectral liens at three different time intervals from the striking of the discharge: solid line, $t=0 \mathrm{~s}$; doted line, $t=30 \mathrm{~s}$; dashed line, $t=60 \mathrm{~s}$. All labels are the same as Fig. 3 .

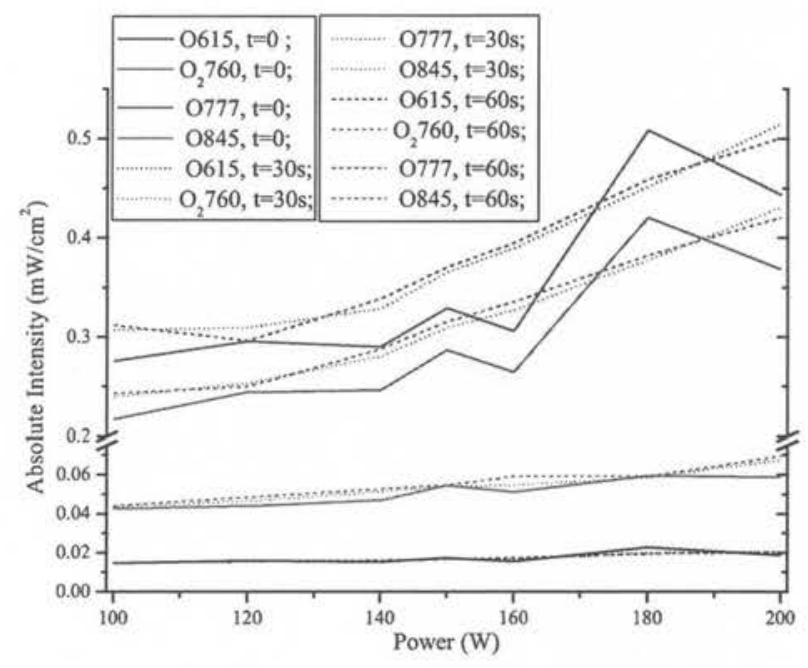

Fig. 6. (Color online) Absolute intensity of oxygen's atomic and molecular spectral emissions at three different time intervals from the striking of the discharge: solid line, $t=0 \mathrm{~s}$; doted line, $t=30 \mathrm{~s}$; dashed line, $t=60 \mathrm{~s}$. All labels are the same as Fig. 3 .

44 emission, as well as other helium spectral emissions, all of ${ }_{45}$ these were observed to increase with processing time and 46 applied $\mathrm{rf}$ power. Figures 6 and 7 show the oxygen and 47 nitrogen spectral emissions, respectively. The O777 and ${ }_{48}$ O845 show (Fig. 6) similar trends to the He706 (Fig. 5) with 49 respect to processing time and applied rf power. The other so two oxygen spectral emissions $\left(\mathrm{O} 615\right.$ and $\left.\mathrm{O}_{2} 706\right)$ do not ${ }_{51}$ Display significant intensity fluctuations with changes in ${ }_{52}$ processing time or applied $\mathrm{rf}$ power. The mechanisms of 53 creation the $\mathrm{O} 615$ and the $\mathrm{O}_{2} 706$ are discussed in Sects. 2.2 ${ }_{54}$ and 2.3 , and it will be more discussed in this section in ${ }_{65}$ conjunctions with the He706 spectral emissions. The absolute 56 spectral emission of molecular nitrogen (Fig. 7) shows 57 oscillation around an average value for each of the three 58 different time intervals. These oscillations are suppressed 59 with the processing time.

Figure 5 shows that the high helium flow rate overrules ${ }_{61}$ nitrogen's importance in a plasma discharge. The nitrogen

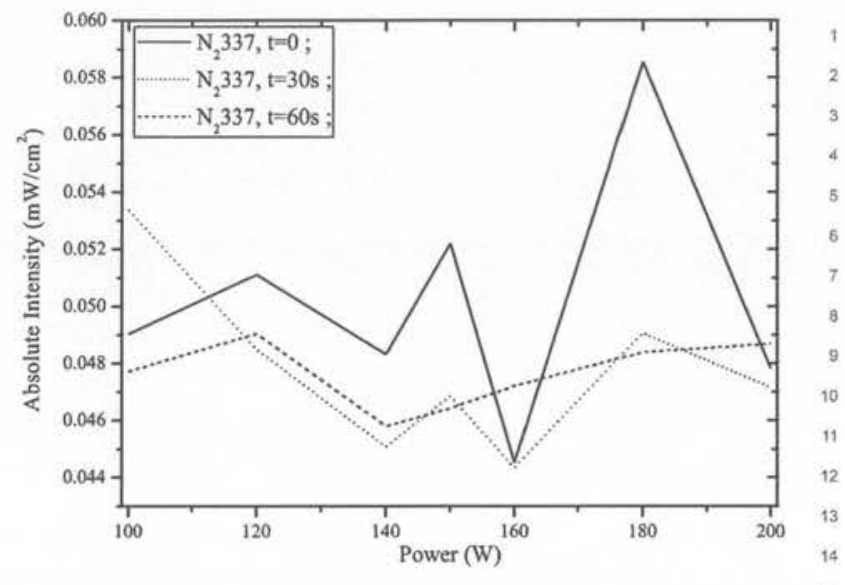

Fig. 7. Absolute spectral intensity of nitrogen molecular band at $337 \mathrm{~nm}$ and at three different time intervals from the striking of the discharge: solid line, $t=0 \mathrm{~s}$; doted line, $t=30 \mathrm{~s}$; dashed line, $t=60 \mathrm{~s}$. All labels are the ${ }^{17}$ same as Fig. 3.

spectral emission is present only through $\mathrm{N}_{2} 337$ (see Fig. 3), 21 which is a unique case since the majority of open air plasma 22 discharges are very rich in nitrogen emission. ${ }^{20}$ ) The $\mathrm{N}_{2} 337{ }_{23}$ $\mathrm{nm}$ emission lines correspond to the following transition 24 $\mathrm{N}_{2}\left(\mathrm{C}^{3} \Pi_{\mathrm{u}}{ }^{+}\right) \rightarrow \mathrm{N}_{2}\left(\mathrm{~B}^{3} \Pi_{\mathrm{g}}{ }^{+}\right){ }^{21)}$ The $\mathrm{N}_{2}\left(\mathrm{C}^{3} \Pi_{\mathrm{u}}{ }^{+}\right)$state can be 25 populated in several ways and has a lifetime of $38 \mathrm{~ns}$. Firstly, 26 it can be populated through the direct electron impact ${ }_{27}$ excitation from the $\mathrm{N}_{2}$ ground state: $\mathrm{N}_{2}\left(\mathrm{X}^{1} \Sigma_{\mathrm{g}}{ }^{+}\right)_{v=0}+e_{\text {fast }} \rightarrow 28$ $\mathrm{N}_{2}\left(\mathrm{C}^{3} \Pi_{\mathrm{u}}^{+}\right)_{v^{\prime}=0,1}+e_{\text {slow, }}$, where the threshold energy of fast 29 electrons equals to $11.5 \mathrm{eV}$. In this case, the population rate 30 for the upper level of the $\mathrm{N}_{2} 337$ transition is proportional to 31 the $\mathrm{N}_{2}$ ground state number density and the density of fast ${ }_{32}$ electrons. A second possible population mechanism of the 33 $\mathrm{N}_{2}\left(\mathrm{C}^{3} \Pi_{\mathrm{u}}{ }^{+}\right)$state is through the electron recombination of ${ }_{34}$ $\mathrm{N}_{2}{ }^{+}\left(\mathrm{X}^{2} \Sigma_{\mathrm{g}}{ }^{+}\right)$followed by decay. The second mechanism is 35 less probable as there is no significant spectral emission 36 recorded for a wavelength of 391-393 nm (Fig. 3). In this ${ }_{37}$ spectral region, nitrogen should has very strong emissions, 38 which are created by the electron transition from $\mathrm{N}_{2}{ }^{+}{ }_{39}$ $\left(\mathrm{B}^{2} \Sigma_{\mathrm{u}}{ }^{+}, v=0\right)$ to $\mathrm{N}_{2}{ }^{+}\left(\mathrm{X}^{2} \Sigma_{\mathrm{u}}{ }^{+}, v^{\prime}=0\right)$ state. The $\mathrm{N}_{2}{ }^{+}{ }_{40}$ $\left(\mathrm{B}^{2} \Sigma_{\mathrm{u}}{ }^{+}\right)$is populated through the Penning reaction and/or 41 the charge transfer from $\mathrm{He}_{2}{ }^{+}$ions. The temporal profile of ${ }_{42}$ the $391-393 \mathrm{~nm}$ line therefore reflects the evolution of the 43 helium metastables and molecular ions. ${ }^{22)}$ Similarly, the 44 helium line at $706 \mathrm{~nm}$ indicates the presence of either 45 energetic electrons or $\mathrm{He}_{2}{ }^{+}$ions and high energy electrons. ${ }^{22)}{ }_{46}$ The absence of a strong/any $\mathrm{N}_{2}{ }^{+}$emission supports the 47 suggestion that there was an insufficient number of helium 48 metastable atoms. The lifetime of electrons at matastable 49 levels should be in the order of $\mu$ s or longer ${ }^{5}$ but, due to its 50 high quenching rates, it is drastically lower. ${ }^{23)}$ These results 51 are similar to those previously reported in Ref. 7, where it 52 was shown that helium metasable atoms were shown to be 53 less important in influencing plasma chemistry as gas flow 54 rates increased. As it is presented in Fig. 5, two helium 55 spectra emissions (He388 and He501), which represent so helium metastable atoms, have the lowest intensity within 57 triplet/singlet helium states. The other four helium lines 58 exhibit much higher intensities and these lines represent the 59 helium atom in its ground state (as discussed earlier). The 60 temporal profiles of all helium spectral lines are similar 61 


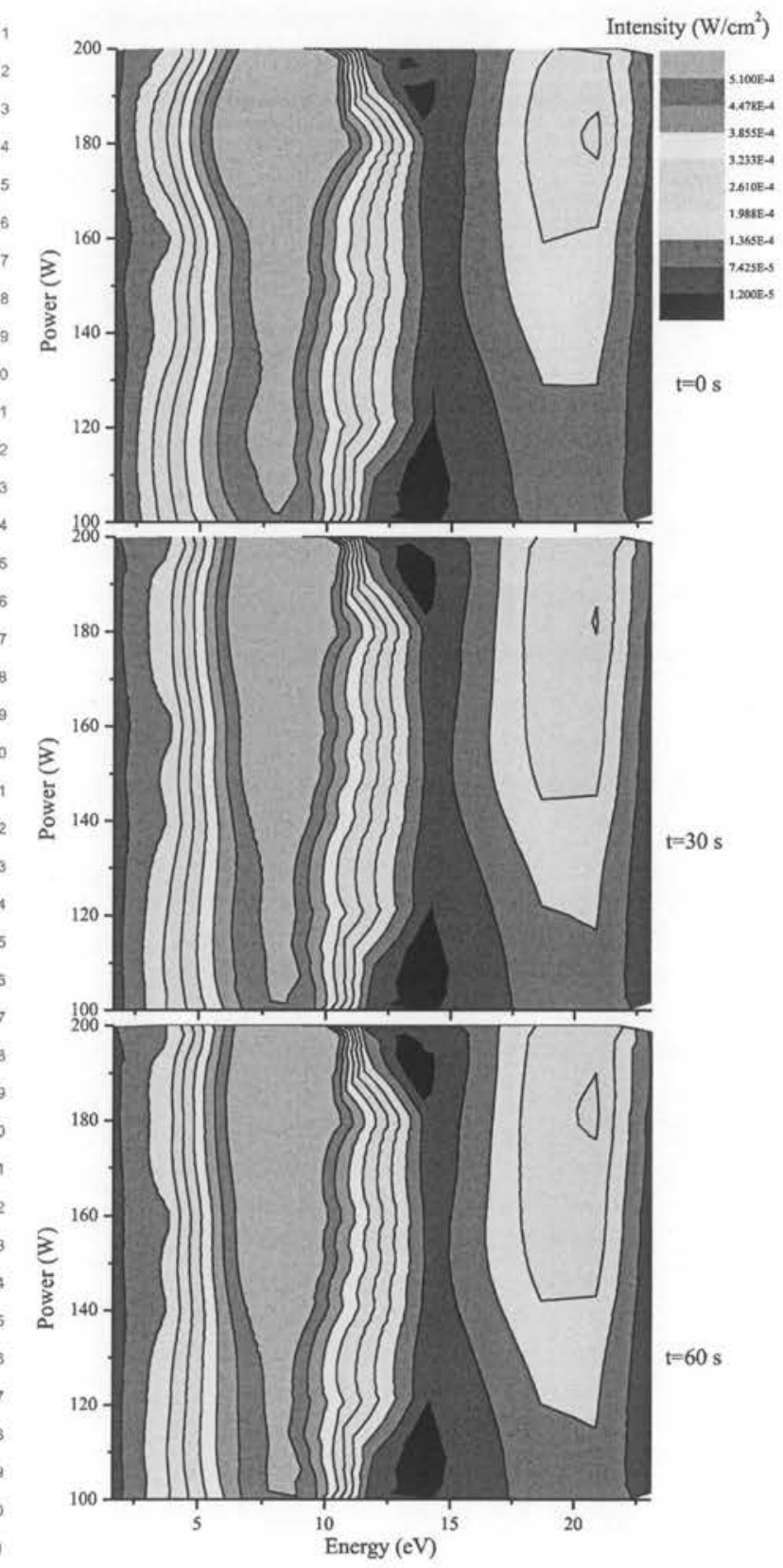

Fig. 8. (Color online) The total spectral intensity for all 13 spectral profiles at three different times $(0,30$, and $60 \mathrm{~s})$ from the ignition of the discharge.

${ }_{47}$ (Fig. 5), and this is a case for all other emissions detailed in ${ }_{48}^{4}$ Fig. 3. Figure 8 shows the absolute spectral intensities as a 49 function of the rf power, processing time and the energy of 50 the upper energy levels.

The spectra's intensities shown in Fig. 8 stay almost 52 constant up to $60 \mathrm{~s}$ time period investigated, which indicates 53 a high degree of plasma stability, and this is clearly very 54 important for any technological application. The difference in ${ }_{55}$ the intensities of the excited plasma species in Fig. 8 were 56 probably related to their different life spans and the energy 57 thresholds. The peak intensity, in Fig. 8 of $8 \mathrm{eV}$, gives an 58 indication of the dynamics of energetic electrons. This 58 suggests that the profiles of excited plasma species may be $s 0$ influenced by both their lifetimes and the dynamics of 61 energetic electrons.

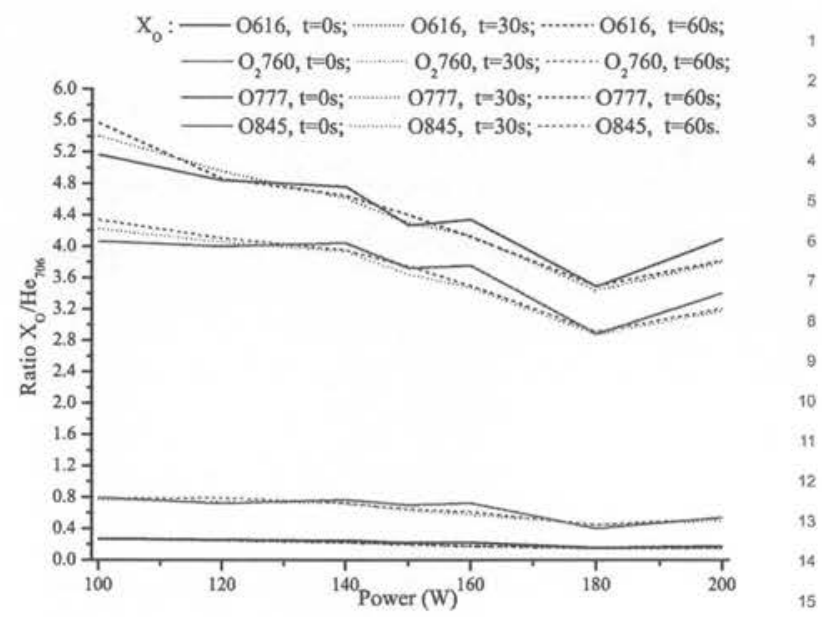

Fig. 9. (Color online) Temporal oxygen spectral emission: solid line, $t=0 \mathrm{~s}$; doted line, $t=30 \mathrm{~s}$; dashed line, $t=60 \mathrm{~s}$.

The optical emission spectrum (Fig. 3) consists of, atomic 20 and molecular oxygen, as well as helium, hydrogen and 21 nitrogen species emissions. Oxygen could come from the 22 ambient air, the $\mathrm{He}-\mathrm{O}_{2}$ gas mixture or from $\mathrm{H}_{2} \mathrm{O}$ dissociation. 23 Electron impact excitation of molecular oxygen, at low 24 collision energies, is of particular importance because of its 25 role in atmospheric physics. Transitions between the $\mathrm{X}^{3} \Sigma_{26}$ ground state and the two lowest $\mathrm{a}^{1} \Delta$, and $\mathrm{b}^{1} \Sigma$ electronically 27 excited states are forbidden by optical dipole selection rules. 28 However the transitions occur as a result of higher-order 29 interactions. The metastable $b^{1} \Sigma$, state is important because ${ }_{30}$ of its long lifetime of $7 \mathrm{~s}^{24}$ ) It also permits the production of ${ }_{31}$ sufficient numbers of atomic oxygen to enable the study of 32 excited molecular species by means of collision scattering 33 experiments. The very long lifetime of the molecular oxygen 34 metastable molecule makes its quenching very efficient. The 35 quenching of singlet molecular oxygen involves the deacti- 36 vation of the excited state of the molecule. ${ }^{10)}$ Deactivation 37 can be accomplished by either physical or chemical 38 quenching. Physical quenching only results in the deactiva- 39 tion of singlet oxygen to its ground state, with no oxygen 40 consumption or product formation. In contrast chemical ${ }_{41}$ quenching results in singlet oxygen reactions with quencher ${ }_{42}$ $\mathrm{R}$ to produce $\mathrm{RO}_{2}$. The two major mechanisms of ${ }^{1} \mathrm{O}_{2}{ }_{43}$ quenching are known to be energy transfer and charge 44 transfer quenching.

Energy transfer quenching is the reverse reaction through 46 which singlet oxygen is formed. ${ }^{25}$ ) It involves formation of ${ }_{47}$ triplet quencher and ground state oxygen $\left({ }^{1} \mathrm{O}_{2}+\mathrm{Q} \rightarrow{ }_{48}\right.$ $\left.{ }^{3} \mathrm{O}_{2}+\mathrm{Q}\right)$.

Charge transfer quenching involves the interaction be- 50 tween the electron-deficient ${ }^{1} \mathrm{O}_{2}$ molecule and electron ${ }_{51}$ donors to form a charge transfer complex. ${ }^{25}$ ) Intersystem 52 crossing restrictions are relaxed in the complex, ${ }^{10)}$ which can ${ }_{53}$ then dissociate into donor and ground state oxygen. The ${ }_{54}$ spectral radiation based on this mechanism includes: $\mathrm{O}_{2} 760,55$ 0615, O777, and 0845 emissions. Figure 9 presents the 56 atomic and molecular oxygen emission in the respect to the 57 He706 emission. It can be observed that the temporal 58 emission profile of the $\mathrm{O} 777$ and $\mathrm{O} 845$ in this figure is 59 different to that of nitrogen and helium. The weak temporal 60 dependence of the $\mathrm{O} 777$ and $\mathrm{O} 845$ emission pattern suggests 61 
1. that $\mathrm{O}\left({ }^{5} \mathrm{P}\right)$ and $\mathrm{O}\left({ }^{3} \mathrm{P}\right)$ are mainly populated by the direct excitation of atoms by electron impact, with a lifetime of 3 upper energy levels of 29 and 32 ns respectively. Under4 pinning that He706 emissions, detected in helium discharges s with a small concentration of impurities, can be used to indicate the presence of energetic electrons. The 0777 and 0845 cannot be created by a dissociative recombination with s the ground state $\mathrm{O}_{2}{ }^{+}$because the rate is strongly peaked toward low energy electrons ${ }^{26}$ ) and there is insufficient energy for these to produce the excited atoms of interest 11 since the difference between the ionization potential of $\mathrm{O}_{2}$ $2(12.07 \mathrm{eV})$ and the bond dissociative energy $(5.09 \mathrm{eV})^{27)}$ is 3 substantially less than the atomic excitation energy $(11 \mathrm{eV})$ ${ }_{14}$ for the $\mathrm{O}\left({ }^{5} \mathrm{P}\right)$ and $\mathrm{O}\left({ }^{3} \mathrm{P}\right)$ states. Another option for the creation 15 of $\mathrm{O} 777$ and $\mathrm{O} 845$ is the dissociative excitation of the 16 molecular oxygen. The emission of $\mathrm{O}_{2} 760$ is almost constant 17 with time and with the rf power, and it does not follow the 18 trends of the $\mathrm{O} 777$ and $\mathrm{O} 845$ emissions (see Fig. 6). The 19 slopes of the $\mathrm{O} 777$ and $\mathrm{O} 845$ are an order of magnitude ${ }_{20}$ greater than the $\mathrm{O}_{2} 760$ slope. The similar trends of these ${ }_{21}$ emissions are also recorded in Fig. 9. It is concluded from 22 this study that the general interpretation that a dissociative 23 molecule oxygen excitation is the dominant mechanism for 24 an 0777 emission, and the atomic oxygen excitation is much 25 more important for an 0845 emission, ${ }^{28)}$ was found not to be 26 the case for a high helium flow rate plasma source. Another ${ }_{27}$ possible channel for creation of $\mathrm{O} 777$ and 0845 could also 28 be radiative cascading from the higher, exited levels in an ${ }_{20}$ oxygen atom. ${ }^{8)}$ The 0615 spectral line has an upper energy so level lifetime of $43 \mathrm{~ns}$, while its lower energy level is the 31 same as the upper energy level of 0777 . The intensity of ${ }_{32} \mathrm{O} 615$ is insensitive to the change of $\mathrm{rf}$ power or the ${ }_{33}$ processing time (Fig. 6). This similar dependence could be ${ }_{34}$ seen in Fig. 9 for this emission. This indicates that the direct 35 excitation of an oxygen atom to a high laying energy level ${ }_{30}\left(4 \mathrm{~d}^{5} \mathrm{D}^{\circ}\right)$ by high energetic electrons, is not important for ${ }_{37}$ creating this (O615) emission. Thus the only mechanism 38 important for the creation of oxygen radicals in the states ${ }_{39}^{3} \mathrm{p}^{5} \mathrm{P}$ and $\mathrm{p}{ }^{5} \mathrm{P}$, in a high flow helium atmospheric plasma 40 discharge, is a direct excitation of atoms by high energetic 41 electrons (the He706 emission pattern). In Fig. 9 the 42 minimum ratio, recorded at $180 \mathrm{~W}$, could be attributed to a 43 change (increase or decrease) in $T_{\mathrm{e}}$ (i.e., EEDF) or to a ${ }_{44}$ change in helium metastable kinetics. Namely, the $\mathrm{X}_{\mathrm{O}} /$ ${ }_{45} \mathrm{He} 706$ increase suggests that the electron energy distribution 46 shifted towards a higher energy value. Electron energy ${ }_{47}$ distribution changes affect the dissociation coefficient of the ${ }_{48}$ oxygen molecule. ${ }^{29)}$ Thus, this electron energy distribution 49 change, induced by the addition of oxygen flow to the fixed 50 helium flow, introduced the change in atomic oxygen in the 51 plasma.

The $\mathrm{OH}$ band $\left(\mathrm{A}^{2} \Sigma^{+}, v=0-\mathrm{X}^{2} \Pi, v^{\prime}=0\right)$ was inves53 tigated and shows a red degradation with main band head at ${ }_{54} 308.986 \mathrm{~nm}^{9)}$ The $\mathrm{OH}$ radicals could be produced through ${ }_{55} \mathrm{H}_{2} \mathrm{O}$ dissociation or/and is produced in the isothermal flow 56 plasma jet by the reaction $\mathrm{H}+\mathrm{NO}_{2} \rightarrow \mathrm{NO}+\mathrm{OH}$, where the ${ }_{57} \mathrm{H}$ atoms are formed by the plasma discharge of highly humid 58 ambient air and helium mixtures. The second channel is 59 mostly unlikely since the high helium flow rate limits the so importance of nitrogen. Since the dissociation of molecules ${ }_{61}\left(\mathrm{H}_{2} \mathrm{O}\right)$ is most likely described by the gas kinetic temperature,

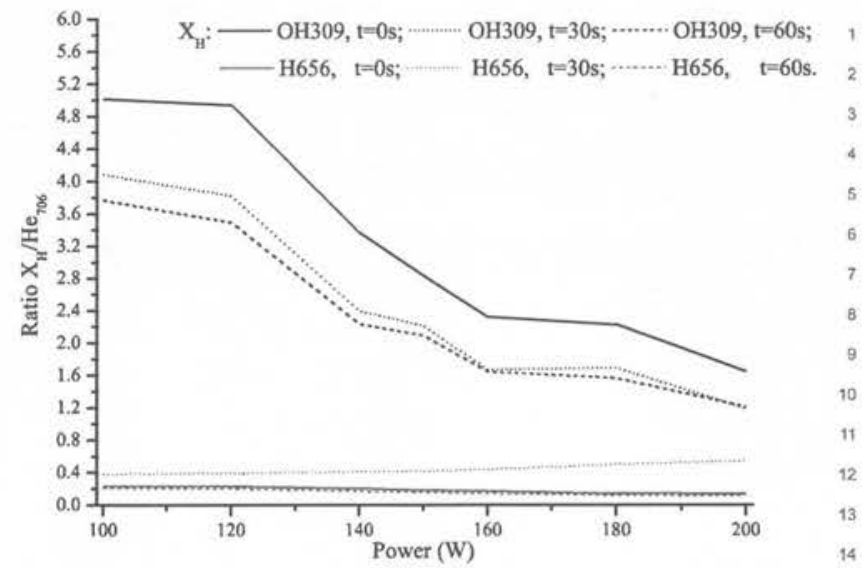

Fig. 10. (Color online) Temporal $\mathrm{OH} 309$ and H656 spectral emissions: a solid line, $t=0 \mathrm{~s}$; a doted line, $t=30 \mathrm{~s}$; a dashed line, $t=60 \mathrm{~s}$.

the radical $\mathrm{OH}$ can be used as an indication of temperature in 20 an ambient gas plasma discharge. ${ }^{30)}$ The $\mathrm{OH} 309$ emission ${ }_{21}$ described in this work is used for gas temperature measure- 22 ment and was benchmarked against infrared thermal imagin- 23 ing $^{12)}$ and the gas temperature was in the range $40-70^{\circ} \mathrm{C}$. ${ }^{24}$ Overall the $\mathrm{OH}$ band $\left(\mathrm{A}^{2} \Sigma^{+}, v=0-\mathrm{X}^{2} \Pi, v^{\prime}=0\right)$ was 25 observed to be very sensitive/accurate for a plasma gas 26 temperature in the range of $300-6000 \mathrm{~K}$. Quantification of 27 $\mathrm{OH}$ radicals in atmospheric pressure plasma jets can be useful ${ }_{28}$ for the understanding of $\mathrm{OH}$ formation mechanisms and 29 plasma generation. $\mathrm{OH}$ radicals exist at a relatively large 30 distance from a main plasma plume of atmospheric pressure ${ }_{31}$ helium plasma jets. ${ }^{11)}$ Figure 10 shows the ratio of both the 32 $\mathrm{OH}$ and $\mathrm{H}$ spectral intensities to $\mathrm{He} 706$.

With increases of $\mathrm{rf}$ power, as well with processing time, 34 the intensities of $\mathrm{OH} 309$ (not shown) has a $\pm 50 \%$ fluctuation 35 around mean value of $0.25 \mathrm{~mW} / \mathrm{cm}^{2}$, during the same time 36 the H656 (not shown) spectral intensity monotonously ${ }_{37}$ increases four times. By dividing these intensities by the 38 He706 emission intensity (which represents high energy 39 electrons) the graph in Fig. 10 was generated. This shows a 40 monotonic decrease in the ratio of the intensities of $\mathrm{OH} 309$ 41 (no intensity fluctuation) and a very small increase in the 42 $\mathrm{H} 656$ signal. The energy required for a $\mathrm{H}_{2} \mathrm{O}$ dissociation ${ }_{43}$ is $5.03 \mathrm{eV}$ and the product of this dissociation is an $\mathrm{OH}_{44}$ molecule and a hydrogen atom. Figure 10 suggests that the 45 electron energy distribution function is shifted towards a 46 higher energy.

\section{Conclusion}

Open air plasma discharges are widely used for surface 50 activation and cleaning. The reactive species created in these 51 discharges could have a significant impact on technology 52 processes and on the environment. Electrical discharges in ${ }_{53}$ ambient air produce some very (potentially) hazardous 54 species such as: $\mathrm{NO}_{x}, \mathrm{OH}^{*}, \mathrm{H}^{*}, \mathrm{O}^{*}, \mathrm{O}_{3}$, and/or $\mathrm{H}_{2} \mathrm{O}_{2}$. From 55 this study it was concluded that helium atmospheric plasma 56 jets, with a high gas flow rates, are more stable (no arcing) 57 and they do not depend on impurities $\left(\mathrm{N}_{2}, \mathrm{O}_{2}, \ldots\right)$ to sustain ${ }_{58}$ the plasma discharge. Nitrogen contamination increases the ${ }_{59}$ gas temperature due to its lower heat conductivity than that of 60 a helium atom. In an optimized helium gas flow, a plasma ${ }_{61}$ 
discharge has no turbulent flow, i.e., the plasma jet is stable 2 and quiet. The large applicator head allows a laminar flow of gas which is well structured, with a hot and bright core. ${ }_{4}$ Moreover, the small amount of $\mathrm{O}_{2}$ added to the plasma forming gas (helium) causes the plasma length to be reduced to couple of millimeters.

In the vast majority of helium discharges in ambient air, a emission spectra clearly show the efficiency of helium to 9 excite the impurities absorbed on electrodes, or existing as traces within an atmosphere. In this work, with a high helium " gas flow rate, the significance of spectral emissions other than the helium atom is observed to be unimportant. 13 Wavelength resolved optical emission profiles suggest that, 14 the helium emission indicates that high energy electrons 15 (spectral emission at $706 \mathrm{~nm}$ ) are more important than helium metastables (spectral emissions at 388 and $501 \mathrm{~nm}$ ), on the overall spectral emission. The lifetime of helium metastables i energy levels are indicated to be drastically reduced as a result of quenching.

The high helium gas flow rate narrows the production channel of the oxygen radicals to the direct electron impact 22 (excitation process) from the ground state of the O I. The 3 general interpretation that the dissociative molecule oxygen ${ }_{24}$ excitation is the dominant mechanism for 0777 emission, 5 and that atomic oxygen excitation is much more important for ${ }_{6}$ O845 emission was found not to be the case for the high $\mathrm{He}-\mathrm{O}_{2}$ flow rate plasma discharge.

Overall the high helium flow rate is used in an attempt to minimize the ratio of gas convection to chemical reaction time scale (recombination). This favors the rapid transport of 1 newly created radicals and excited species to the surface 2 under treatment. The addition of a low levels $(<3 \%)$ of an 3 electronegative gas (oxygen) to helium gas causes a reduction 4 of the electron density and consequently, a reduction of the electrical conductivity. This also causes an increase in the sustaining voltage and consequently, in the electric field 7 strength. Therefore the mean electron kinetic energy is $s$ increased, and this leads to an increase of the excitation temperature.

\section{Acknowledgments}

This material is based upon works supported by the ${ }_{43}$ Science Foundation Ireland under Grant No. 08/SRC/I1411 44 as well as the "Fresh-Pack" project funded by the National 45 Development Plan, through the Food Institutional Research 46 Measure, administered by the Department of Agriculture, ${ }_{47}$ Food and the Marine, Ireland. V. Milosavljević is also grateful to the Ministry of Education and Science of the 4 Republic of Serbia under Grant No. OI171006. *vm@dit.ie

1) Y. Ohguchi and K. Takahama, J. Phys. Soc. Jpn. 55, 3889 (1986).

2) S. Yamada, H. Yoshimura, and M. Tachibana, J. Phys. Soc. Jpn. 79, 054708 (2010).

3) C. Yubero, M. D. Calzada, and M. C. Garcia, J. Phys. Soc. Jpn. 74, ${ }^{4}$ 2249 (2005).

4) R. Brandenburg, J. Ehlbeck, M. Stieber, T. v. Woedtke, J. Zeymer, O. Schlüter, and K.-D. Weltmann, Contrib. Plasma Phys. 47, 72 (2007).

5) Yu. Ralchenko, A. E. Kramida, J. Reader, and NIST ASD Team, NIST Atomic Spectra Database (ver. 4.1.0) (National Institute of Standards ${ }^{8}$ and Technology, Gaithersburg, MD, 2013) [http://physics.nist.gov/asd]. 9

6) V. Milosavljević, D. Popović, and A. R. Ellingboe, J. Phys. Soc. Jpn. 10 78, 084501 (2009).

7) K. Tachibana, Y. Kishimoto, and O. Sakai, J. Appl. Phys. 97, 123301 (2005).

8) V. Milosavljević, A. R. Ellingboe, and S. Daniels, Eur. Phys. J. D 64, ${ }^{13}$ 437 (2011).

9) G. F. R. S. Herzberg, Molecular Spectra and Molecular Structure I. 15 Spectra of Diatomic Molecules (D. van Nostrand, Princeton, NJ, 1950) 16 2nd ed.

10) S. Popović, M. Nikolić, J. Upardhyay, and L. Vučković, AIAA 2010- ${ }^{17}$ 5042 (2010).

11) S. Pellerin, J. M. Cormier, F. Richard, K. Musiol, and J. Chapelle, ${ }^{19}$ J. Phys. D 29, 726 (1996).

12) M. Donegan, V. Milosavljević, and D. P. Dowling, Plasma Chem. Plasma Process. 33, 941 (2013).

13) S. E. Babayan and R. F. Hicks, US Patent 7329608 (2008).

14) D. P. Dowling, F. T. O'Neill, S. J. Langlais, and V. J. Law, Plasma ${ }^{23}$ Processes Polym. 8, 718 (2011).

15) K.-D. Weltmann, E. Kindel, R. Brandenburg, C. Meyer, R. Bussiahn, 25 C. Wilke, and T. von Woedtke, Contrib. Plasma Phys. 49, 631 (2009). ${ }_{26}$

16) R. J. Zaldivar, J. Nokes, G. L. Steckel, H. I. Kim, and B. A. Morgan, 27 J. Compos. Mater. 44, 137 (2010).

17) R. J. Zaldivar, J. Salfity, G. Steckel, B. Morgan, D. Patel, J. P. Nokes, ${ }^{28}$ and H. I. Kim, J. Compos. Mater. 46, 1925 (2012).

18) C. E. Nwankire, V. J. Law, A. Nindrayog, B. Twomey, K. Niemi, V, 30 Milosavljević, W. G. Graham, and D. P. Dowling, Plasma Chem. 31 Plasma Process. 30, 537 (2010).

19) N. K. Bibinov, A. A. Fateev, and K. Wiesemann, J. Phys. D 34, $1819^{32}$ (2001).

20) J. L. Walsh, J. J. Shi, and M. G. Kong, Appl. Phys. Lett. 89, $161505^{34}$ (2006).

21) A. Qayyum, S. Zeb, M. A. Naveed, S. A. Ghauri, M. Zakaullah, and A. 36 Waheed, J. Appl. Phys. 98, 103303 (2005).

22) G. Nersisyan and W. G. Graham, Plasma Sources Sci. Technol. 13, $582^{37}$ (2004).

23) S. Suzuki, H. Itoh, H. Sekizawa, and N. Ikuta, J. Phys. Soc. Jpn. 62, 39 2692 (1993).

24) T. G. Slanger and R. A. Copeland, Chem. Rev. 103, 4731 (2003).

25) B. Halliwell and M. C. John, Free Radical in Biology and Medicine (Clarendon, Oxford, U.K., 1982) 2nd ed.

26) M. A. Biondi, in Principles of Laser Plasmas, ed. G. Bekefi (Wiley, ${ }^{43}$ New York, 1976) Chap. 4.

27) K. P. Huber and G. Herzberg, Constants of Diatomic Molecules (Van 45 Nostrand Reinhold, New York, 1979).

28) R. E. Walkup, K. L. Saenger, and G. S. Selwyn, J. Chem. Phys. 84, ${ }_{47}^{46}$ 2668 (1986).

29) H. S. W. Massey, Electronic and Ionic Impact Phenomena (Clarendon, ${ }^{48}$ Oxford, U.K., 1969) Vol. II.

30) S. Tsurubuchi, T. Iwai, and T. Horie, J. Phys. Soc. Jpn. 36, 537 (1974). 50 